\title{
Oxygen uptake, heart rate and activities of locomotor muscles during a critical swimming speed protocol in the gilthead sea bream Sparus aurata
}

\author{
Hachim Mouniboudine ${ }^{1}$, Rouyer Tristan ${ }^{2}$, Dutto Gilbert ${ }^{3}$, Kerzerho Vincent ${ }^{4}$, Bernard Serge ${ }^{4}$, \\ Bourjea Jerome ${ }^{2}$, McKenzie David ${ }^{1,}{ }^{*}$
}

${ }^{1}$ Marbec Université Montpellier, CNRS, Ifremer, IRD Montpellier, France

2 Marbec Université Montpellier, Ifremer, CNRS, IRD Sète, France

3 Ifremer, Laboratoire SEA, Chemin de Maguelonne Palavas-Les-Flots, France

${ }^{4}$ LIRMM Université Montpellier, CNRS Montpellier, France

* Corresponding author : David McKenzie, email address : david.mckenzie@cnrs.fr

\begin{abstract}
:
Oxygen uptake, heart rate, and contraction frequencies of slow oxidative (SO) and fast glycolytic (FG) muscle, were measured simultaneously in gilthead seabream Sparus aurata submitted to stepwise increases in current speed in a swimming respirometer. Variation in oxygen uptake was closely related to variation in heart rate, over initial steps these rose in concert with an increase in contraction frequency of SO muscle. There was an asymptote in oxygen uptake and heart rate at high speeds, that reflected a transition from exclusive use of aerobic SO muscle to a combination of SO and anaerobic FG muscle, and which preceded fatigue.
\end{abstract}


The critical swimming speed $\left(\mathrm{U}_{\text {crit }}\right)$ protocol (Brett, 1964) is a well-established method to measure exercise performance in fishes (Beamish, 1978; Webb, 1998; McKenzie \& Claireaux, 2010). It has been used widely to investigate effects on performance of environmental factors such as temperature or salinity (Randall \& Brauner, 1991; Farrell et al., 2008; McKenzie \& Claireaux, 2010) or as a biomarker of toxicological effects of aquatic pollutants (e.g. Beaumont et al., 1995; McKenzie et al., 2007; Wood et al., 1996), and has had important applications in research for conservation of valuable migratory species (Farrell et al., 2008; Eliason et al., 2011). The protocol exposes fish to stepwise increases in speed in a swimming respirometer, until fatigue. The fish swims against the current by rheotactic reflex, holding position in the swim channel of the respirometer.

In fishes that use body-caudal fin locomotion (Webb, 1998), slow-twitch oxidative (SO) 'red' muscle is used to power swimming at low to intermediate swimming speeds. Strips of muscle along the flanks beat the tail at steady frequencies directly proportional to swimming speed and the fish maintains a relatively static position in the respirometer. Contraction of SO muscle relies on ATP generated aerobically, it is well vascularized and supplied with nutrients and oxygen in the blood, pumped by the heart. So, increased aerobic swimming speed is linked to marked increases in oxygen uptake rate $\left(\mathrm{M}_{02}\right)$ and heart beat frequency $\left(f_{\mathrm{H}}\right)$ (Chatelier et al., 2005; McKenzie \& Claireaux, 2010). At a certain high current speed in a $U_{\text {crit }}$ test, however, the fish starts to engage its large myotomal blocks of fast-twitch glycolytic (FG) 'white' muscle, with irregular powerful tailbeats. These propel the fish forward in the swim channel, after which they drift back on the current, a 'burst and coast' swimming mode (Webb, 1998). The FG muscle is poorly vascularised and uses endogenous fuel stores to generate ATP anaerobically so, when it is recruited at high swim speeds, there can be an asymptote or even slight decline in $\mathrm{M}_{02}$ and $f_{\mathrm{H}}$ (Beamish, 1978; Lee et al., 2003; Chatelier et al., 2005). This may be linked to reduced rates of SO muscle contraction but this has not been explicitly investigated. Once fishes recruit FG muscle and engage burst and coast swimming, they typically fatigue quite rapidly.

Although patterns of SO and FG muscle recruitment have been studied during forced swimming in fishes (Roberts \& Graham, 1979; Rome et al., 1985; Rome \& Alexander, 1990; Geist et al., 2003) no study has, to the best of our knowledge, simultaneously measured $\mathrm{M}_{02}, f_{\mathrm{H}}$ and muscle activity during a $U_{\text {crit }}$ trial. This is interesting better to understand how patterns of oxygen uptake and heart rate relate to sequential recruitment of aerobic oxidative and then anaerobic glycolytic muscle. Specifically, to demonstrate that an asymptote in $f_{\mathrm{H}}$ and $\mathrm{M}_{\mathrm{O} 2}$ at high speeds coincides with recruitment of FG muscle, and to ascertain what SO muscle activity patterns are at the highest speeds prior to fatigue.

Experiments were performed on gilthead seabream Sparus aurata L. 1758, a coastal marine teleost that is highly prized and widely farmed in the Mediterranean. It uses a sub- 
carangiform swimming mode and, in the wild, adults can migrate over hundreds of kilometres (Lasserre, 1976). Experimental procedures were approved by the ethics committee for animal experimentation $\mathrm{n}^{\circ} 036$ of the French Ministère de l'Enseignement Superieur, de la Recherche et de l'Innovation, with reference number APAFIS\#10130-201704071516523 v3.

Six S. aurata with a mean $( \pm$ SD) mass of $612 \pm 10 \mathrm{~g}$ and forklength of $294 \pm 13 \mathrm{~mm}$ were studied, from a population of animals obtained from Cannes Aquaculture fish farm and maintained at Ifremer Experimental Aquaculture Station at Palavas-les-Flots, in outdoor $3000 \mathrm{l}$ tanks provided with a flow of local seawater at prevailing seasonal temperatures and an average salinity over the study period of 34\%o. Fish were held for over 18 months prior to experiments, fed commercial pellets daily but fasted for $24 \mathrm{~h}$ prior to trials. Experiments were in April and May, when seawater temperature was 15 to $17^{\circ} \mathrm{C}$.

Fish were anesthetized by immersion in $0.1 \mathrm{~g} \mathrm{l}^{-1}$ benzocaine in aerated seawater, until active ventilation ceased, then positioned on an operating table with gills irrigated with aerated seawater containing $0.05 \mathrm{~g} \mathrm{l}^{-1}$ benzocaine. Insulated stranded stainless steel wire electrodes (0.05 $\mathrm{mm}$ wire diameter, $0.23 \mathrm{~mm}$ total diameter; Steel 7 Strand, A-M Systems, www.phymep.com), $800 \mathrm{~mm}$ total length, insulated to within $0.5 \mathrm{~cm}$ of the tip, were placed to measure ECG and EMGs. For ECG, two electrodes were inserted on either side of the animal, under the cleithrum behind the $4^{\text {th }}$ gill arch in close proximity to the heart. For EMGs, pairs of electrodes were inserted on one flank, along the axis of the animal beginning at the insertion of the dorsal fin and at a distance of $4 \mathrm{~cm}$ from each other; for SO muscle superficially just above midline (avoiding any contact with the lateral line); for FG muscle into epaxial muscle dorsally. The position of electrodes in SO and FG muscle was confirmed by dissection post mortem. All wires were held in place with sutures at point of insertion, then gathered and held in place with a common suture in front of the dorsal fin, so they trailed above fish during swimming trials (Blasco et al., 2016). After surgery, fish were recovered in a Steffensen-type swim-tunnel respirometer (vol. $49 \mathrm{l}$ ) for at least $24 \mathrm{~h}$ in aerated seawater at $16^{\circ} \mathrm{C}$, swimming in a current equivalent to 0.5 body lengths per second (BL s-1).

The respirometer is designed to provide a non-turbulent water flow with a uniform velocity profile, in which to exercise fish at controlled current speeds while measuring their rates of oxygen uptake (McKenzie et al., 2001). The anterior portion of the swim section was shielded with black plastic sheeting to avoid visual disturbance of fish, which spontaneously occupied this area. After recovery, seabream were exposed to progressive increments of swimming speed of $0.5 \mathrm{BL} \mathrm{s}^{-1}$ each $40 \mathrm{~min}$, until fatigue. Fish were considered fatigued when they rested their caudal fins on the downstream grid for at least 10s. Critical swimming speed ( $\mathrm{U}_{\text {crit, }}$ in BL s${ }^{-1}$ ) was calculated as described by Brett (1964). The $\mathrm{Mo}_{2}$ was measured in mmol $\mathrm{O}_{2}$ $\mathrm{kg}^{-1} \mathrm{~h}^{-1}$ by respirometry twice at each speed by cyclical "intermittent stopped-flow" (Steffensen, 
1989; Svendsen et al., 2016), using the Aquaresp program (University of Copenhagen, www.aquaresp.com). Briefly, the cycle alternates two phases; firstly, the swim tunnel receives no water and the fish consumes oxygen within the closed system, measured by an optical sensor (Firesting 02, Pyro-Science, www.pyro-science.com) and associated software (Pyro Oxygen Logger), with data taken into Aquaresp. Secondly, Aquaresp starts a pump by USB relay (Cleware, www.cleware-shop.de) that flushes aerated seawater through the tunnel, so renewing oxygen and removing wastes. Each cycle was $20 \mathrm{~min}, 8$ of measurement and 12 of flushing; two cycles were completed at each swimming speed. Standard metabolic rate (SMR; basal metabolic rate at acclimation temperature) was estimated by back-extrapolating the relationship between $\mathrm{MO}_{2}$ and speed to a notional speed of zero (Brett, 1964; Chabot et al., 2016). Active Metabolic Rate (AMR; maximal $\mathrm{MO}_{2}$ at acclimation temperature) was identified during swimming and usually occurred at speeds approaching $U_{\text {crit }}$ (McKenzie et al., 2003). Absolute aerobic scope (AAS) was the net difference between AMR and SMR, factorial scope (FAS) was AMR/SMR (Beamish, 1978).

To record $f_{\mathrm{H}}$, (beats $\left.\mathrm{min}^{-1}\right)$ and frequencies of $\mathrm{SO}$ and $\mathrm{FG}$ muscle contractions $\left(f_{\mathrm{so}}\right.$ and $f_{\mathrm{FG}}$, respectively, in Hz), ECG and EMG electrodes were connected to a BIOPAC MP36R data acquisition system linked to a computer with BIOPAC Acqknowledge software (www.biopac.com). ECG signals were.... EMG signals were filtered and recorded using the Acqknowledge 'EMG 30-1000 Hz' acquisition package, which sampled at $2000 \mathrm{~Hz}$ with low band pass at $1000 \mathrm{~Hz}$ and high band pass at $30 \mathrm{~Hz}$. Signals were displayed on Acqknowledge throughout trials, then recorded twice for $5 \mathrm{~min}$ at each swimming speed. The $f_{\mathrm{H}}$ was calculated based on mean time required for $10 \mathrm{R}-\mathrm{R}$ intervals of ECG waveforms. The $f_{\mathrm{SO}}$ and $f_{\mathrm{FG}}$ were obtained from the interval between 5 EMG burst onsets, at each speed.

Data were tested for normality using Shapiro-Wilk Test prior to parametric tests. The effects of swimming speed on $\mathrm{M}_{\mathrm{O} 2}, f_{\mathrm{H}}, f_{\mathrm{SO}}$ and $f_{\mathrm{FG}}$ were assessed by one way-analysis of variance (ANOVA) for repeated measures. When significant effects were observed, Bonferroni post-hoc tests were used to compare means. For descriptive purposes of patterns in mean data during the stages of the $U_{\text {crit }}$ trial, linear or exponential regressions were applied, as detailed below. The relationship of $f_{\mathrm{H}}$ to $\mathrm{M}_{02}$ was described by linear regression. All analyses were performed with SigmaStat 4.0 (Systat Software Inc., www. systatsoftware.com).

Mean ( $\pm \mathrm{SE}) \mathrm{U}_{\text {crit }}$ was $3.2 \pm 0.2 \mathrm{BL} \mathrm{s}^{-1}$, all animals completed at least 20 min swimming at a speed of $3 \mathrm{BL} \mathrm{s}^{-1}$, so data were collected for all variables for speeds of 0.5 to $3 \mathrm{BL} \mathrm{s}^{-1}$ (Fig 1). Mean $\mathrm{M}_{02}$ increased significantly $(\mathrm{P}<0.001$ by ANOVA) with swimming speed, steeply until $2 \mathrm{BL}$ $\mathrm{s}^{-1}$ followed by an asymptote at higher speeds (fig 1A). Application of an exponential relationship to mean data prior to the asymptote, namely $0.5 \mathrm{BL} \mathrm{s}^{-1}$ to $2 \mathrm{BL} \mathrm{s}^{-1}$, revealed a high correlation coefficient $\left(\mathrm{R}^{2}=0.994\right)$. Mean SMR was $3.37 \pm 0.28 \mathrm{mmol} \mathrm{O}_{2} \mathrm{~kg}^{-1} \mathrm{~h}^{-1}$, mean AMR was 
$11.45 \pm 0.60 \mathrm{mmol} \mathrm{O}_{2} \mathrm{~kg}^{-1} \mathrm{~h}^{-1}$ and occurred at $2.83 \pm 0.14 \mathrm{BL} \mathrm{s}^{-1}$. The AAS was $8.07 \pm 0.49 \mathrm{mmol}$ $\mathrm{O}_{2} \mathrm{~kg}^{-1} \mathrm{~h}^{-1}$ and FAS $3.52 \pm 0.29$. Mean $f_{\mathrm{H}}$ increased significantly $(\mathrm{P}<0.001$ by ANOVA) with swimming speed, this was exponential between 0.5 and $2 \mathrm{BL} \mathrm{s}^{-1}\left(\mathrm{R}^{2}=0.996\right)$ with evidence of an asymptote at speeds beyond, coinciding with the asymptote in $\mathrm{MO}_{2}$ (fig 1B). Maximum $f_{\mathrm{H}}$ was 96 \pm 4 beats $\mathrm{min}^{-1}$, at an average swimming speed of $2.92 \pm 0.19 \mathrm{BL} \mathrm{s}^{-1}$. There was a highly significant linear relationship between $f_{\mathrm{H}}$ and $\mathrm{MO}_{2}$ (fig 2) whereby $\mathrm{MO}_{2}=0.208\left(f_{\mathrm{H}}\right)-5.648\left(\mathrm{R}^{2}=\right.$ 0.987, $\mathrm{P}<0.0001$ ). The reciprocal relationship was used to estimate mean $f_{\mathrm{H}}$ at mean SMR, being 50 beats min $^{-1}$ at $3.37 \mathrm{mmol} \mathrm{O}_{2} \mathrm{~kg}^{-1} \mathrm{~h}^{-1}$, such that average factorial cardiac scope was 1.9 $(96 / 50)$.

Fig. S1 in Supplementary Material shows representative traces of EMG signals for SO and FG muscle activity, such as were used to calculate their contraction frequency based upon intervals between burst onsets. There was a significant $(\mathrm{P}<0.001$ by ANOVA) increase in mean $f_{\text {so }}$ up to $2 \mathrm{BL} \mathrm{s}^{-1}$ after which contraction frequency dropped and, at $3 \mathrm{BL} \mathrm{s}^{-1}$, it was significantly lower than at $2.5 \mathrm{BL} \mathrm{s}^{-1}$ (fig $1 \mathrm{C}$ ). Among fish, the maximum $f_{\text {so }}$ of $4.00 \pm 0.31 \mathrm{~Hz}$ occurred at a mean speed of $2.67 \pm 0.2 \mathrm{BL} \mathrm{s}^{-1}$, providing a mean stridelength (BL swum per SO muscle contraction) of $0.69 \pm 0.07 \mathrm{BL}$. By contrast, FG muscle had no contractile activity until a speed of $2 \mathrm{BL} \mathrm{s}^{-1}$, after which it increased rapidly and significantly $(\mathrm{P}<0.001$ by ANOVA) to reach a maximum of $1.07 \pm 0.30 \mathrm{~Hz}$ at $3 \mathrm{BLs}^{-1}$, the last swimming speed that all fish achieved prior to fatigue (fig 1D). The engagement of FG muscle was associated with a gait transition to a "burst and coast' swimming mode, where sea bream used irregular powerful tailbeats that 'burst' it forward in the swim channel, after which it 'coasted' back on the current until repeating the action.

The results demonstrate how patterns of $\mathrm{M}_{02}$ and $f_{\mathrm{H}}$ during a $\mathrm{U}_{\text {crit }}$ protocol relate to sequential recruitment of aerobic oxidative and anaerobic glycolytic muscle in a teleost with sub-carangiform locomotion. The $U_{\text {crit }}$ values are similar to reports for farmed $S$. aurata (Basaran et al., 2007; Svendsen et al., 2015), comparisons are confounded by differences in body size and water temperature, which both affect $U_{\text {crit }}$ performance (Beamish, 1978; McKenzie \& Claireaux, 2010). The changes in $\mathrm{M}_{02}$ with swimming speed, comprising an initial exponential increase followed by an asymptote, have been reported in various species (Chatelier et al., 2005; Lee et al., 2003; Tudorache et al., 2015; this study), although others report an exponential increase up until fatigue (McKenzie et al., 2003; Steinhausen et al., 2005; Methling et al., 2011; Tudorache et al., 2015). The FAS during $U_{\text {crit }}$, approximately 3.5 , is less than a previous report of around 5 (Svendsen et al., 2015), which may be due to factors such as fish size and rearing conditions. The $f_{\mathrm{H}}$ at low speeds, and as estimated at SMR from the linear relationship between $f_{\mathrm{H}}$ and $\mathrm{M}_{\mathrm{O} 2}$, were similar to a previous reports of 'routine' $f_{\mathrm{H}}$ for $S$. aurata at this temperature (Aissaoui et al., 2000). 
The exponential increase in $\mathrm{M}_{\mathrm{O2}}$ and $f_{\mathrm{H}}$ during initial stages of the $\mathrm{U}_{\text {crit }}$ was clearly a response to increasing oxygen and nutrient demands of mitochondria in working SO muscle (Teulier et al., 2019), required to contract the muscle and beat the tail faster as speed steps were imposed. It is well-established that $\mathrm{M}_{02}$ rises exponentially with speed of aerobic swimming in fishes (Beamish, 1978; Webb, 1998). The estimated stridelength of SO muscle, approximately 0.7 BL per tailbeat, is typical for teleosts with sub-carangiform locomotion (Beamish, 1978). The asymptote in $\mathrm{M}_{\mathrm{O} 2}$ and $f_{\mathrm{H}}$ at $2 \mathrm{BL} \mathrm{s}^{-1}$ coincided with $f_{\mathrm{So}}$ also reaching an asymptote coupled with engagement of FG muscle. The fact that SO muscle continued to contract at relatively high frequencies, alongside recruitment of the large, powerful FG muscle blocks, may explain why $\mathrm{M}_{02}$ and $f_{\mathrm{H}}$ did not decline but essentially remained stable up until fatigue at $\mathrm{U}_{\text {crit. }}$. Although $\mathrm{FG}$ muscle is poorly vascularised, it does receive some blood flow and is a very large organ, representing up to $70 \%$ of the mass of the seabream (Teulier et al., 2019). The very close coupling of $\mathrm{M}_{\mathrm{O} 2}$ to $f_{\mathrm{H}}$, throughout the $\mathrm{U}_{\text {crit }}$ protocol, was confirmed by the fact that variation in $f_{\mathrm{H}}$ explained almost $99 \%$ of variation in $\mathrm{M}_{02}$.

The $f_{\mathrm{FG}}$ was much lower than $f_{\mathrm{So}}$, with much more variation around the mean because contractions were aperiodic. It has been suggested that fatigue in a $U_{\text {crit }}$ swim test is in fact a behavioural response, that occurs when a fish cannot engage the full power of FG muscle in the limited confines of the swim tunnel, so 'chooses' to fall back against the rear screen (Peake \& Farrell, 2006; Tudorache et al., 2007).

In conclusion, the data provide the first simultaneous measure of $\mathrm{M}_{\mathrm{O} 2}, f_{\mathrm{H}}$ and contraction frequencies of $\mathrm{SO}$ and $\mathrm{FG}$ muscle in a fish during a $\mathrm{U}_{\text {crit }}$ protocol. The results demonstrate unequivocally that variation in $\mathrm{M}_{\mathrm{O} 2}$ is closely related to variation in $f_{\mathrm{H}}$ and that an asymptote in $\mathrm{M}_{02}$ and $f_{\mathrm{H}}$, at high speeds, reflects a transition from exclusive use of aerobic SO muscle to a combination of SO and anaerobic FG muscle.

\section{Acknowledgements}

This research was part of the MERLIN-POPSTAR project funded by Ifremer. Authors thank Michel Prinet (LIENSS La Rochelle) and Aurélien Lledo (Ifremer Palavas) for technical help with the swim tunnel, and Laurent Deknyff (LIRMM) for fashioning ECG and EMG electrodes.

\section{References}

Aissaoui, A., Tort, L., \& Altimiras, J. (2000). Circadian Heart Rate Changes and Light-Dependence in the Mediterranean Seabream Sparus Aurata. Fish Physiology and Biochemistry, 22, 89-94. Basaran, F., Ozbilgin, H., \& Ozbilgin, Y. D. (2007). Comparison of the Swimming Performance of Farmed and Wild Gilthead Sea Bream, Sparus Aurata. Aquaculture Research, 38, 452-456. Beamish, F. W. H. (1978). Swimming Capacity. In Fish Physiology Volume 7 (Hoar, W. S., Randall, D. J., eds), pp. 101-187 New York: Academic Press. 
Beaumont, M., Butler, P., \& Taylor, E. (1995). Plasma Ammonia Concentration in Brown Trout in Soft Acidic Water and Its Relationship to Decreased Swimming Performance. The Journal of experimental biology, 198, 2213-2220.

Blasco, F. R., McKenzie, D. J., Taylor, E. W., \& Rantin, F. T. (2016). The Role of the Autonomic Nervous System in Control of Cardiac and Air-Breathing Responses to Sustained Aerobic Exercise in the African Sharptooth Catfish Clarias Gariepinus. Comparative biochemistry and physiology. Part A, Molecular \& integrative physiology, 203, 273-280.

Brett, J. R. (1964). The Respiratory Metabolism and Swimming Performance of Young Sockeye Salmon. Journal of the Fisheries Research Board of Canada, 21, 1183-1226.

Chabot, D., Steffensen, J. F., \& Farrell, A. P. (2016). The Determination of Standard Metabolic Rate in Fishes. Journal of Fish Biology, 88, 81-121.

Chatelier, A., McKenzie, D. J., \& Claireaux, G. (2005). Effects of Changes in Water Salinity upon Exercise and Cardiac Performance in the European Seabass (Dicentrarchus Labrax). Marine Biology, 147, 855-862.

Eliason, E. J., Clark, T. D., Hague, M. J., Hanson, L. M., Gallagher, Z. S., Jeffries, K. M., ... Farrell, A. P. (2011). Differences in Thermal Tolerance among Sockeye Salmon Populations. Science (New York, N.Y.), 332, 109-112.

Farrell, A. P., Hinch, S. G., Cooke, S. J., Patterson, D. A., Crossin, G. T., Lapointe, M., \& Mathes, M. T. (2008). Pacific Salmon in Hot Water: Applying Aerobic Scope Models and Biotelemetry to Predict the Success of Spawning Migrations. Physiological and Biochemical Zoology, 81, 697-708.

Geist, D. R., Brown, R. S., Cullinan, V. I., Mesa, M. G., Vanderkooi, S. P., \& McKinstry, C. A. (2003). Relationships between Metabolic Rate, Muscle Electromyograms and Swim Performance of Adult Chinook Salmon. Journal of Fish Biology, 63, 970-989.

Lasserre, G. (1976). Dynamique Des Populations Ichtyologiques Lagunaires - Application a Sparus aurata L., Université des Sciences et Techniques du Languedoc, Montpellier.

Lee, C. G., Farrell, A. P., Lotto, A., MacNutt, M. J., Hinch, S. G., \& Healey, M. C. (2003). The Effect of Temperature on Swimming Performance and Oxygen Consumption in Adult Sockeye (Oncorhynchus Nerka) and Coho (O. Kisutch) Salmon Stocks. Journal of Experimental Biology, 206, 3239-3251.

McKenzie, D. J., Cataldi, E., Romano, P., Owen, S. F., Taylor, E. W., \& Bronzi, P. (2001). Effects of Acclimation to Brackish Water on the Growth, Respiratory Metabolism, and Swimming Performance of Young-of-the-Year Adriatic Sturgeon (Acipenser Naccarii). Canadian Journal of Fisheries and Aquatic Sciences, 58, 1104-1112.

McKenzie, D. J., \& Claireaux, G. (2010). The Effects of Environmental Factors on the Physiology of Aerobic Exercise. In Fish Locomotion: An Eco-ethological Perspective (Domenici, P., Kapoor, 
B. G., eds), pp. 308-344 Boca Raton: CRC Press.

McKenzie, D. J., Martinez, R., Morales, a., Acosta, J., Morales, R., Taylor, E. W., ... Estrada, M. P.

(2003). Effects of Growth Hormone Transgenesis on Metabolic Rate, Exercise Performance and Hypoxia Tolerance in Tilapia Hybrids. Journal of Fish Biology, 63, 398-409.

McKenzie, D. J., Garofalo, E., Winter, M. J., Ceradini, S., Verweij, F., Day, N., ... Taylor, E. W. (2007). Complex Physiological Traits as Biomarkers of the Sub-Lethal Toxicological Effects of Pollutant Exposure in Fishes. Philosophical transactions of the Royal Society of London. Series B, Biological sciences, 362, 2043-2059.

Methling, C., Tudorache, C., Skov, P. V., \& Steffensen, J. F. (2011). Pop up Satellite Tags Impair Swimming Performance and Energetics of the European Eel (Anguilla Anguilla). PLOS ONE, 6.

Peake, S. J., \& Farrell, A. P. (2006). Fatigue Is a Behavioural Response in Respirometer-Confined Smallmouth Bass. Journal of fish biology, 68, 1742-1755.

Randall, D. J., \& Brauner, C. J. (1991). Effects of Environmental Factors on Exercise in Fish. Journal of Experimental Biology, 126, 113-126.

Roberts, J. L., \& Graham, J. B. (1979). Effect of Swimming Speed on the Excess Temperatures and Activities of Heart and Red and White Muscles in the Mackerel, Scomber Japonicus. Fishery Bulletin, 76, 861-867.

Rome, L. C., \& Alexander, R. M. (1990). The Influence of Temperature on Muscle Velocity and Sustained Performance in Swimming Carp. Journal of experimental biology, 154, 163-178.

Rome, L. C., Loughna, P. T., Goldspink, G., \& others. (1985). Temperature Acclimation: Improved Sustained Swimming Performance in Carp at Low Temperatures. Science (New York, NY), $228,194$.

Steffensen, J. F. (1989). Some Errors in Respirometry of Water Breathers: How to Avoid and Correct for Them. Fish Physiology and Biochemistry, 6, 49-59.

Steinhausen, M. F., Steffensen, J. F., \& Andersen, N. G. (2005). Tail Beat Frequency as a Predictor of Swimming Speed and Oxygen Consumption of Saithe (Pollachius Virens) and Whiting (Merlangius Merlangus) during Forced Swimming. Marine Biology, 148, 197-204.

Svendsen, J. C., Tirsgaard, B., Cordero, G. A., \& Steffensen, J. F. (2015). Intraspecific Variation in Aerobic and Anaerobic Locomotion: Gilthead Sea Bream (Sparus Aurata) and Trinidadian Guppy (Poecilia Reticulata) Do Not Exhibit a Trade-off between Maximum Sustained Swimming Speed and Minimum Cost of Transport. Frontiers in Physiology, 6, 43.

Svendsen, M. B. S., Bushnell, P. G., \& Steffensen, J. F. (2016). Design and Setup of IntermittentFlow Respirometry System for Aquatic Organisms. Journal of Fish Biology, 88, 26-50.

Teulier, L., Thoral, E., Queiros, Q., McKenzie, D. J., Roussel, D., Dutto, G., ... Saraux, C. (2019). Muscle Bioenergetics of Two Emblematic Mediterranean Fish Species: Sardina Pilchardus 
and Sparus Aurata. Comparative Biochemistry and Physiology Part A: Molecular \& Integrative Physiology, 235, 174-179.

Tudorache, C., Viaenen, P., Blust, R., \& De Boeck, G. (2007). Longer Flumes Increase Critical Swimming Speeds by Increasing Burst-Glide Swimming Duration in Carp Cyprinus Carpio, L. Journal of Fish Biology, 71, 1630-1638.

Tudorache, C., Burgerhout, E., Brittijn, S., \& van den Thillart, G. (2015). Comparison of Swimming Capacity and Energetics of Migratory European Eel (Anguilla Anguilla) and New Zealand Short-Finned Eel (A. Australis). Frontiers in Physiology, 6, 256.

Webb, P. W. (1998). Swimming. In The Physiology of Fishes (Evans, D. D., ed), pp. 1-38 Boca Raton: CRC Press.

Wood, a W., Johnston, B. D., Farrell, a P., \& Kennedy, C. J. (1996). Effects of Didecyldimethylammonium Chloride (DDAC) on the Swimming Performance, Gill Morphology, Disease Resistance, and Biochemistry of Rainbow Trout ( Oncorhynchus Mykiss ). Canadian Journal of Fisheries and Aquatic Sciences, 53, 2424-2432. 


\section{Figure legends}

Figure 1. Effects of swimming speed, in bodylengths $\mathrm{s}^{-1}$, on mean $( \pm S E M)$ oxygen uptake rate (A); heart rate (B), and the contraction frequencies of slow-twitch oxidative (SO) and fast-twitch glycolytic (FG) muscles ( $C$ and $D$, respectively), in $\mathrm{n}=6$ Sparus aurata submitted to a critical swimming speed protocol. On each panel, similar letters indicate no significant difference in the mean, by 1-way ANOVA for repeated samples and Bonferonni post-hoc test $(\mathrm{P}>0.05)$.

Figure 2. Least squares linear relationship between mean heart beat frequency $\left(f_{\mathrm{H}}\right)$ and mean oxygen uptake rate $\left(\mathrm{M}_{02}\right)$ in $\mathrm{n}=6$ Sparus aurata submitted to a critical swimming speed protocol. The line is described by the equation $\mathrm{M}_{02}=0.208\left(f_{\mathrm{H}}\right)-5.648\left(\mathrm{R}^{2}=0.986\right)$. 
Figure 2


Heart rate (beats $\min ^{-1}$ )
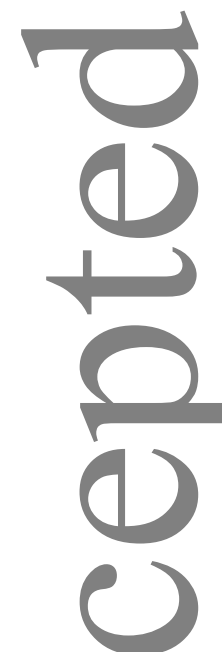

J

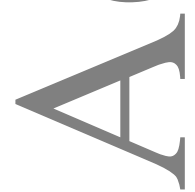

This article is protected by copyright. All rights reserved. 